\title{
Escape Rooms for Learning: A Systematic Review
}

Panagiotis Fotaris ${ }^{1}$, Theodoros Mastoras ${ }^{2}$

${ }^{1}$ School of Computing, Engineering and Mathematics, University of Brighton, Brighton, UK.

${ }^{2}$ Department of Applied Informatics, University of Macedonia, Thessaloniki, Greece.

p.fotaris@brighton.ac.uk

mastoras@uom.edu.gr

\begin{abstract}
Following the recent shift from traditional didactic classroom models to the adoption of active learning approaches, escape rooms and breakout games are increasingly being used in academia as a method for experiential, peer-group, game-based learning. Although they have the potential to enable new forms of teaching and transform the learning experience, escape rooms are a relatively new concept and there is not a substantial amount of work exploring their tendencies, affordances, and challenges on education. This paper addresses the lack of empirical evidence on the impact of escape rooms on educational settings by presenting a systematic review of 68 peer-reviewed studies published in scientific journals and conference proceedings between 2009 and April 2019. To analyse and critically appraise the current state of knowledge and practice in educational escape rooms, it considers aspects such as fields of education, target audience, game type and location, time limit, team size, and study results. The systematic review also highlights the advantages and challenges of these new learning activities, as well as their positive impact on student motivation and soft skills development. The analysis indicates that educational escape rooms can provide an enjoyable experience that immerses students as active participants in the learning environment. Additionally, they give learners the opportunity to engage in an activity that rewards teamwork, creativity, decision-making, leadership, communication, and critical thinking. Although instructional design for educational escape rooms is complex and time consuming, once the game has been developed it can be further applied in successive years. The results of this work aim to lay the groundwork for educators and other stakeholders by offering new insights with effective advice and recommendations for the successful incorporation of escape rooms into their teaching strategies.
\end{abstract}

Keywords: escape room, breakout game, game-based learning, systematic review, gamification

\section{Introduction}

As 'Digital Natives', students of today think and process information differently, thus providing a challenge to their teachers who often experience difficulties in keeping them motivated and engaged through conventional taught learning. There is an acute need for educational innovations and ideas to make a meaningful impact on the students' educational experience (Serdyukov, 2017). Therefore, traditional education is giving way to the use of approaches that have reportedly sustained high levels of motivation and engagement in the classroom, such as integrating educational content within game-based context. Findings of independent experiments performed in secondary and higher education settings showed that students who were subjects to learning with video games reported significant improvements in subject understanding, diligence, and motivation (Fotaris et al., 2016). Additionally, Barata et al. (2013) suggest that students who have taken part in a gamified learning course have increased attendance rates, report higher levels of enjoyment and find learning more interesting.

Game-based learning (GBL) takes advantage of gaming technologies and techniques to create a fun, motivating, and interactive virtual learning environment that promotes situated experiential learning. Unlike the one-sizefits-all lectures, GBL can be balanced to suit the learners' skill-level in order to prevent them from becoming frustrated or bored and to allow them to experience flow, a state of optimal experience for learning (Csikszentmihalyi, 1990). Although GBL also comprises non-digital examples, an overwhelming proportion of GBL research has been devoted to digital GBL, a field that aims at integrating educational elements into computer games and/or digital simulations. While researchers have agreed on the potential of such solutions for both instructional teaching and the exploration of new topics (Giang et al., 2018), most digital educational games are individual and do not develop team building or group communication (Dietrich, 2018). Nevertheless, research into the design, development, and evaluation of non-digital GBL applications still remains scarcer than research surrounding their digital counterparts (Clarke et al., 2017). However, this trend is beginning to change, mainly due to the rising popularity of escape rooms and their adoption in educational settings which allows to implement more tangible and human-centred activities. 


\section{Background}

Borrowing elements from point-and-click adventure games, live-action role-playing, interactive theatre, treasure hunts, movies, and TV shows, an escape room is "a live-action team-based game where players discover clues, solve puzzles, and accomplish tasks in one or more rooms in order to accomplish a specific goal (usually escaping from the room) in a limited amount of time" (Nicholson 2015). Despite the name, it is not universal for the theme to be escape-based. Players maybe called upon to solve a crime, save a fictional character or find something. Escape rooms are one type of escape games, which are narrative-based challenges that use puzzles, tasks, and a time limit. Other types include puzzlehunts, breakout boxes, escape books, Augmented/Virtual Reality (AR/VR) escape rooms, or portable escape-rooms-in-a-box where most of the puzzles are contained in a box so that players can have the same immersive and challenging experience in the comfort of their home.

Despite being a relatively new concept, escape rooms have become immensely popular in recent years. They were firstly used in Japan in 2007 and grew rapidly in the last 5 years, exploding from 2,800 rooms throughout the world in 2015 to over 7,200 in 2018 (Kroski, 2019). From a pedagogical point of view, escape rooms are based on a social-constructivist approach (Vygotsky, 1978). Learners construct their own knowledge based on real-time experiences of advancing through several challenges in the escape room; they are called to face new and often complex problems, which can be solved by interacting with their peers and getting support from their tutor. The latter can play the role of Game Designer, structuring the learning environment and/or the role of Game Master, providing instructional scaffolding to the learners by facilitating the students' interaction with the material and with each other (Giang et al., 2018).

With that in mind, it may come as no surprise that escape rooms have begun to gain traction in academia. However, it is not feasible or legal to lock a subset of a class in a room and wait until they puzzle their way out. As a compromise, many escape rooms designed for the classroom have been stripped back to a group table-top activity involving a series of locked boxes (Schaffhauser, 2017). While this type of activity loses the complete immersion of a true escape room, it can still provide a motivating and educationally beneficial experience for students when designed appropriately (Clarke et al., 2017). Academically focused escape-style scenarios were popularised by Breakout EDU, an immersive learning games platform founded in 2015 that provides escape room kits for instructors (Rouse, 2017). Educational institutions and libraries have started to integrate this initiative into their programmes (Walsh and Spence, 2018) or to develop their own educational escape rooms (López-Pernas et al., 2019). An educational escape room/game can be defined as "an instructional method requiring learners to participate in collaborative playful activities explicitly designed for domain knowledge acquisition or skill development so that they can accomplish a specific goal (e.g., escape from a physical room or break into a box) by solving puzzles linked to unambiguous learning objectives in a limited amount of time". Having a shared environment for players to work together on a game designed around specific learning outcomes sets the groundwork for active learning.

\section{Motivation and rationale for the study}

The last 3 years have seen the development of an increasing number of educational escape rooms with satisfying results, such as improved communication, collaboration, engagement, and student satisfaction (Friedrich et al., 2018). However, due to their novelty, studies on empirical work or the educational significance of such activities are still limited. Literature about their efficacy and usefulness as an educational tool appears to be very sparse and a systematic review on educational escape rooms has yet to be published (Giang et al., 2018; Sanchez \& Plumettaz-Sieber, 2019). The present study addresses this issue by being the first systematic review that performs a synthesis of the relevant research on educational escape rooms published from 2009 to April 2019 in order to identify and illustrate the opportunities they offer as an instructional tool. Within this context, the research questions that guided this study were the following:

- RQ1: What are the trends of educational escape rooms?

- RQ2: What are the main characteristics of an educational escape room?

- RQ3: What are the advantages and challenges of using escape rooms in educational settings?

\section{Research methodology}

The guidelines proposed by Kitchenham (2007) were adapted for the purposes of this systematic review using the following steps: Planning; Conducting the review; and Reporting the review. Analysis of the results and the 
discussion of findings, trends and conclusions regarding the Preferred Reporting Items for Systematic Reviews and Meta-Analyses (PRISMA) statement recommendation (Moher et al., 2009).

\subsection{Planning}

This initial step was used to identify the most relevant literature to answer the research questions. For that reason, we accomplished an iterative double check focused on peer-reviewed journals indexed in the Social Sciences Citation Index ( $\mathrm{SSCl}$ ) database, and peer-reviewed conference proceedings indexed in the Conference Proceedings Citation Index-Science (CPCl-S) database. The search process spanned from December 2018 to April 2019 and was conducted using electronic databases of authoritative academic resources and publishers, including Scopus, Web of Science, Science Direct, Google Scholar, and ERIC. The inclusion and exclusion criteria are presented in Table 1.

Table 1: Inclusion / exclusion criteria

\begin{tabular}{ll}
\hline Inclusion criteria & Exclusion criteria \\
\hline Studies Publisher between 2009 and 2019 & Non-English language articles \\
Articles published in peer-reviewed journals or & Studies not providing sufficient data for effect size \\
full-length articles published in International & calculation or not having clear summarisation or \\
Conference / Workshop Proceedings & aggregative findings from their qualitative data \\
Studies providing evidence of educational & Articles not presenting evaluation or empirical data (e.g., \\
potential based on a research method & theoretical articles, essays, tool demonstration etc.) \\
Studies describing the use of escape rooms for & Studies not identified as "articles" (e.g., books, book \\
educational purposes & reviews/chapters, editorial publication information) \\
\hline
\end{tabular}

Escape rooms are sometimes known as escape games, room escape games, exit games, breakout games etc. Currently there is no consensus on what to call this type of games, therefore the search keywords included terms and variations for escape rooms in conjunction with terms for possible outcomes, impacts or effects of playing escape games for learning. For example, a query used in Scopus is the following:

("room escape" OR "escape room*" OR "escape game*" OR "game escape" OR "breakout EDU" OR "breakout game*" OR "breakout box" OR "exit game") AND (learn* OR class* OR teach* OR train* OR educat*)

Finally, the following categories for analysis and data coding were defined to assist grouping of all relevant studies based on their shared characteristics: target group; subject area; advantages/challenges of educational escape rooms.

\subsection{Conducting and reporting the review}

After removing the duplicate records, the search procedure yielded 169 results. These articles were initially screened based on their abstract; they were then carefully read by both authors to identify their suitability for the study. The quality of the collected literature was subsequently assessed using the following rigorous quantitative/qualitative rules (Pellas et al., 2018):

- Appropriateness of the research methods and analysis of the study results;

- In-depth illustration of the followed methodology;

- Sufficient presentation of the findings;

- Adequacy of the research questions;

- Alignment of the study findings with the research questions.

Based on the combination of the above rules, 68 of the 169 retrieved articles were identified as relevant to the purpose of this review and were selected for this study (Fig. 1). 46 of them were published in international journals (67.6\%) and 22 in conference proceedings (32.4\%). The data extraction and data synthesis were carried out by reading these 68 papers thoroughly; a data extraction spreadsheet was designed to store the following elements: study name, year and type of publication, target audience, field of education, team size, location, time limit, game type, theme, briefing, debriefing, research questions, evaluation methods, sample size, advantages, challenges, and main findings. 


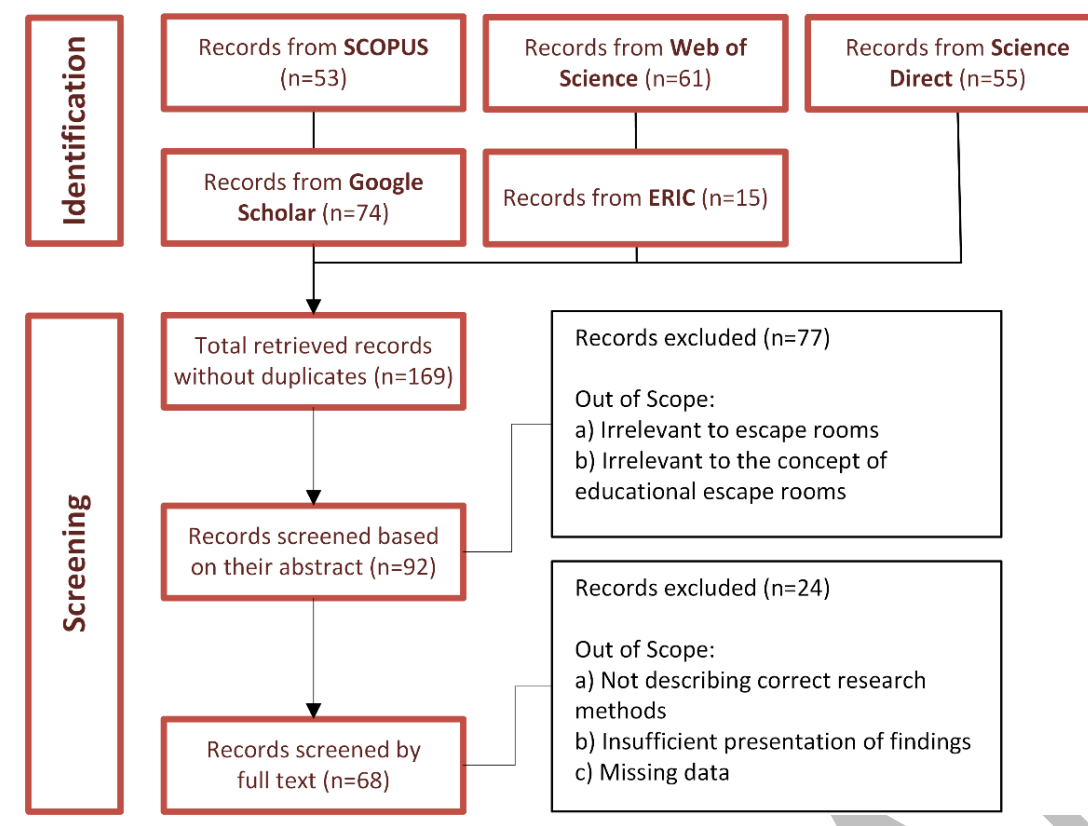

Figure 1. Flowchart for the article selection process

Due to the diversity of the research methods used for instructional design, samples, and data collection, it was not possible to undertake an accurate meta-analysis. However, the overall results were synthesised to extract the main themes under which the findings of the review are identified and presented. To assess interrater reliability with respect to the quality coding of the papers, all papers were coded independently by the two authors. The interrater reliability (IRR) for the total scores was attained using Cohen's kappa (Cohen, 1968); this value was 0.91, showing good agreement between the two authors. Any disagreements were resolved by consensus. The final stage included the analysis of the results and the discussion of findings that answered the research questions of the study.

\section{Results}

\subsection{Trends of educational escape rooms}

Evolution over time: Of the 68 studies selected for the systematic review, 1 study was published in 2009 (1.5\%), 3 in 2016 (4.4\%), 15 in 2017 (22.1\%), 25 in 2018 (36.8\%), and 24 were published by the end of April 2019 (35.3\%) (Fig. 2). Assuming that the publication rate will remain the same throughout 2019 , the projected number of publications for 2019 should be around 72, which is almost three times the total number of publications for 2018. The search indicates that studies are being published at an increasing rate on a yearly basis with no signs of slowing down. It is interesting to note that although the first paper on educational escape games was published in 2009 (Hsu et al.), new articles on this topic were not published before 2016, which is around the time escape rooms began to gain momentum in popularity as a social entertainment activity.

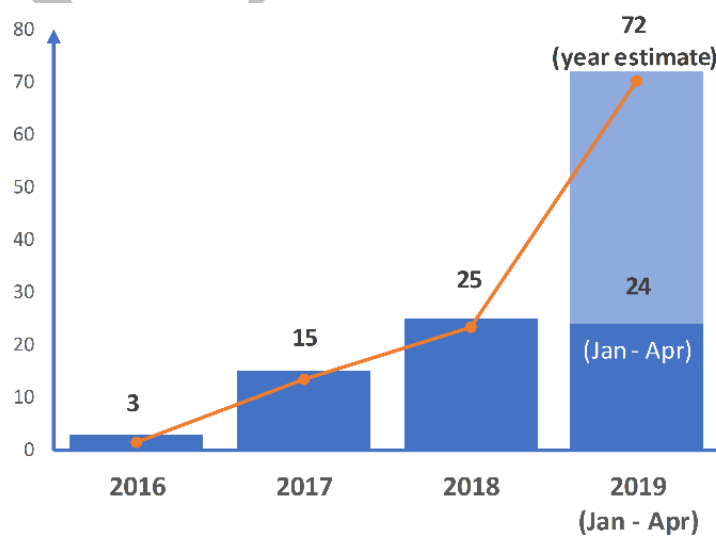

\begin{tabular}{lrr}
\hline Year & $\begin{array}{r}\text { Number } \\
\text { of studies }\end{array}$ & Percentage (\%) \\
\hline 2009 & 1 & $1.5 \%$ \\
$\ldots$ & 0 & $0 \%$ \\
2016 & 3 & $4.4 \%$ \\
2017 & 15 & $22.1 \%$ \\
2018 & 25 & $36.8 \%$ \\
2019 (Jan - Apr) & 24 & $35.3 \%$ \\
\hline
\end{tabular}

Fig. 2: Studies related to the application of escape rooms in education per year 
Target audience: The target audience for most studies was higher education students (72.1\%), followed by secondary (11.8\%) and primary education students (7.3\%), the general public (7.3\%), and staff members (1.5\%) (Table 2). This can possibly be attributed to the fact that universities usually have better resources and more research-focused staff compared to schools and are therefore more likely to lead the way in high quality research. However, based on the eagerness with which schoolteachers have adopted escape rooms in their classes as evidenced by the popularity of Breakout EDU (Rouse, 2017), these numbers may soon change.

Table 2: Analysed studies per target audience

\begin{tabular}{lcr}
\hline Target audience & Studies & \multicolumn{1}{c}{$\%$} \\
\hline Higher Education & 49 & $72.1 \%$ \\
Secondary Education & 8 & $11.8 \%$ \\
Primary Education & 5 & $7.3 \%$ \\
General public & 5 & $7.3 \%$ \\
Staff & 1 & $1.5 \%$ \\
\hline
\end{tabular}

Fields of education: To identify the domain of the educational escape rooms, our classification was based on the broad fields of education proposed by the International Standard Classification of Education ISCED (UNESCO, 2013). Collected data in Table 3 show that most educational escape rooms cover subjects related to the broad field of Health and welfare (29.4\%), focusing on Nursing (Hermanns et al., 2017) and Medicine (Zhang et al., 2019). Natural sciences, mathematics and statistics come second (22.1\%), with Chemistry being the most popular topic in this field (Peleg et al., 2019). 19.1\% of the studies focused on Social sciences, journalism and information, including several studies about Library orientation (Walsh and Spence, 2018), which is an emerging topic for educational escape rooms. Information and Communication Technologies (14.7\%) are represented by escape rooms covering topics such as information literacy (Pun, 2017), programming (López-Pernas et al., 2019), computer networks (Borego et al., 2017), and cryptography (Ho, 2018).

Table 3: Percentage of analysed studies per field of education

\begin{tabular}{lcc}
\hline Broad field & Studies & $\%$ \\
\hline Health and welfare & 20 & $29.4 \%$ \\
Natural Sciences, mathematics and statistics & 15 & $22.1 \%$ \\
Social sciences, journalism and information & 13 & $19.1 \%$ \\
Information and communication technologies & 10 & $14.7 \%$ \\
Generic programmes and qualifications & 5 & $7.4 \%$ \\
Arts and humanities & 4 & $5.8 \%$ \\
Business, administration and law & 1 & $1.5 \%$ \\
\hline
\end{tabular}

\subsection{Main characteristics of educational escape rooms}

Game type: Since their inception, escape rooms were created to exist in physical, digital, or mixed realities (i.e., a new environment in which physical and digital objects coexist). Mainly due to the relatively low cost of props (e.g., locks, boxes, paper-based puzzles, etc.) and faster development time, $76.5 \%$ of the escape rooms in the selected studies are physical, i.e., using only physical objects (e.g., Friedrich et al., 2018). 13.2\% are hybrid, offering both physical (e.g., locks, boxes etc.) and virtual objects (e.g., QR codes, social media platforms etc.) that contained puzzle clues (e.g., Hermanns et al., 2017). Finally, 10.6\% are digital games (e.g., Guo et al., 2017). Although physical escape rooms are by far the most popular type of escape rooms, hybrid escape rooms using $A R / V R$ are expected to increase once these technologies become more mature.

Location: Results in Table 5 reveal that $78.9 \%$ of the escape games took place in the classroom or in a lab. From a logistical point of view this might be a direct consequence of the space limitations often addressed in educational institutions, which prevent an escape room from having its own dedicated and permanent space, thus requiring the game to be portable or quick and easy to set up and take down (Sundsbø, K. 2019). Moreover, when an escape game is played in the classroom students are not required to travel to a different location to participate. Library buildings and hospital rooms are the second and third most common locations, respectively, which correlates with the aforementioned findings about the popularity of escape rooms focusing on Library orientation, Nursing, and Medicine. Other locations include a whole campus with puzzles being spread out over several departments (Mac Gregor, 2018), a commercial escape room, and a factory building. 
Table 4: Analysed studies per game type

\begin{tabular}{lcc}
\hline Game type & Studies & $\%$ \\
\hline Physical & 52 & $76.5 \%$ \\
| Room: 23 (44.2\%) & & \\
| Breakout box: 22 (42.3\%) & & \\
| Other: 7 (13.5\%) & & \\
Hybrid & 9 & $13.2 \%$ \\
Digital & 7 & $10.6 \%$ \\
\hline
\end{tabular}

Table 5: Analysed studies per escape room location

\begin{tabular}{lcr}
\hline Escape Room Location & Studies & $\%$ \\
\hline Classroom / laboratory & 52 & $78.9 \%$ \\
Library building & 7 & $10.6 \%$ \\
Simulated hospital room & 2 & $3.0 \%$ \\
Outdoors & 2 & $3.0 \%$ \\
Campus & 1 & $1.5 \%$ \\
Commercial Escape Room & 1 & $1.5 \%$ \\
Factory building & 1 & $1.5 \%$ \\
\hline
\end{tabular}

Time limit: 52 out of the 68 reviewed studies provided information about the escape room's time limit. As shown in Table 6, most participants had 15-120 minutes to complete the game, excluding any additional time spent for briefing and debriefing activities. The only exceptions were a breakout game with no time limit (Bartlett and Anderson, 2019) and an escape game running over the course of 3 weeks (Mac Gregor, 2018). 78.8\% of the escape rooms lasted one hour or less, which supports previous findings (López-Pernas et al., 2019). The majority of studies (36.5\%) used the 60-minute time limit typically found in commercial escape rooms, as this allows for a sufficient number of puzzles to be used, offers ample time for students to work as a team, and fits into one hour of instruction. In most of these cases escape room activities usually took place during two-hour sessions that also allowed for briefing/debriefing sessions to be conducted. $30.8 \%$ of the reviewed escape rooms had a time limit of 30-50 minutes and $11.5 \%$ lasted $20-25$ minutes. These shorter time limits can be attributed to time constraints; when the available time is restricted to one hour, games have to be shorter to include briefing/debriefing sessions or to be reset if the activity has to be conducted in multiple time slots for large groups (Walsh and Spence, 2018). Additionally, shorter games require less development time. Games lasting 75120 minutes were less frequent (17.3\%) because - besides the obvious logistical issues - they can result in student fatigue (Hsu et al., 2009). However, longer games can give tutors the opportunity to use more meaningful challenges that require more time and effort to be solved (López-Pernas et al., 2019).

Table 6: Analysed studies per time limit

\begin{tabular}{lcc}
\hline Time limit & Studies & $\%$ \\
\hline $15-25 \mathrm{~min}$ & 6 & $11.5 \%$ \\
$30-50 \mathrm{~min}$ & 16 & $30.8 \%$ \\
$60 \mathrm{~min}$ & 19 & $36.5 \%$ \\
$75-120 \mathrm{~min}$ & 9 & $17.3 \%$ \\
3 weeks & 1 & $1.9 \%$ \\
Unlimited & 1 & $1.9 \%$ \\
\hline
\end{tabular}

Table 7: Analysed studies per team size

\begin{tabular}{lcr}
\hline Team size & Studies & \multicolumn{1}{c}{$\%$} \\
\hline 1 & 5 & $9.8 \%$ \\
$2-4$ & 8 & $15.7 \%$ \\
5 & 14 & $27.5 \%$ \\
6 & 11 & $21.6 \%$ \\
$7-10$ & 10 & $19.6 \%$ \\
$10-14$ & 2 & $3.9 \%$ \\
Any & 1 & $2.0 \%$ \\
\hline
\end{tabular}

Team size: This refers to the number of participants in the escape room. Besides 5 studies about single-player digital escape rooms (9.8\%), team size in all other studies ranged from 2 to 14 players, with the exception of an escape game ran in a campus that had no team size limitation (Mac Gregor, 2018). Results in Table 7 reveal that most teams had 5 players $(27.5 \%)$ or more. Compared to the average team size of 4.58 people for commercial escape rooms (Nicholson, 2015), teams in educational escape rooms tend to be bigger. This is mainly due to constraints imposed by classroom size, time, and facilities. Conducting an escape room activity with large cohorts means that several sessions have to take place, which can be a logistical nightmare. As a result, team size compromises often have to be made, which can affect student participation (Adams et al., 2018).

\subsection{Advantages and challenges of using escape rooms in educational environments}

All 68 studies reported at least one advantage, but the majority of them listed more. As expected, educational escape rooms were found to promote teamwork and collaboration (41.2\%) (Ho, 2018), as well as produce high levels of enjoyment (38.3\%) (Peleg et al., 2019) and engagement (32.4\%) (Zhang et al., 2019). Students reported learning gain (30.9\%) (Walsh and Spence, 2018) and increased motivation $(29.4 \%)$ as they found learning through play more interesting. Another common advantage was social interaction and communication (27.9\%); the escape room activity reinforced and strengthened social relationships, which helped establish a sense of belonging. Improved analytical skills such as critical thinking (Adams et al., 2018), problem-solving (16.2\%) and creativity (Foster and Warwick, 2018) (10.3\%) were also signalled as a major advantage (16.2\%), while natural leadership behaviours have reportedly emerged in several studies (10.3\%). Finally, the results show that escape 
rooms can be reused several times with different groups (10.3\%) and provide a supplemental method for reviewing material (10.3\%) (Kinio et al., 2017).

48 of the selected studies (70.6\%) reported some challenges when using escape rooms in educational settings. The most reported challenge refers to poor evaluation (33.8\%); the majority of studies lacked a control group and resorted to surveys addressing the students' perception of the escape room and how it impacted their learning of the concept. This could produce biased results as it was sometimes difficult to obtain a sufficient number of responses. Even in cases where experimental design or pre-post tests were used the results could not lead to safe conclusions due to small sample sizes. Time commitment was also an issue (25\%), as the intellectual and physical labour involved in the creation of an escape room make it a labour-intensive and high resource process that may not appeal to many educators. Other reported challenges include small sample sizes (20.6\%) and limited resources such as room or facilitator availability (14.7\%). Due to time constraints, poor design, or limited playtesting, occasionally games had unbalanced difficulty (11.8\%) and felt too short/too long (10.3\%). Involving students in the game creation to ensure age- and developmentally-appropriate puzzles could address this problem. Timing issues were also reported (8.8\%); having a few minutes to set the game when running events to tight schedules was often stressful for staff. Although educational escape rooms can be cheap to build, budget limitations were still reported (7.4\%). Finally, large group sizes presented logistical challenges, as it was difficult for instructors to conduct the activity in multiple time slots or for participants to deeply engage with the tasks (5.9\%).

Table 8: Advantages of educational escape rooms

\begin{tabular}{lcc}
\hline Advantages & Studies & $\%$ \\
\hline Teamwork/collaboration & 28 & 41.2 \\
Enjoyment & 26 & 38.3 \\
Engagement & 22 & 32.4 \\
Learning gain & 21 & 30.9 \\
Motivation & 20 & 29.4 \\
Social interaction & 19 & 27.9 \\
Critical thinking/problem-solving & 11 & 16.2 \\
Leadership & 7 & 10.3 \\
Creativity & 7 & 10.3 \\
Reusability & 7 & 10.3 \\
Revision method & 7 & 10.3 \\
\hline
\end{tabular}

Table 9: Challenges of educational escape rooms

\begin{tabular}{lcc}
\hline Challenges & Studies & $\%$ \\
\hline Poor evaluation & 23 & 33.8 \\
Time commitment & 17 & 25.0 \\
Small sample size & 14 & 20.6 \\
Limited resources & 10 & 14.7 \\
Unbalanced difficulty & 8 & 11.8 \\
Game length & 7 & 10.3 \\
Timing issues & 6 & 8.8 \\
Budget limitations & 5 & 7.4 \\
Large group size & 4 & 5.9 \\
\hline
\end{tabular}

\section{Discussion and conclusion}

This work presents a systematic literature review of 68 studies focused on educational escape rooms. Results seem to indicate that escape rooms are innovative, active, collaborative and constructivist instructional approaches that can shape learning more powerfully than conventional teaching. They help learners understand the value of seeing problems from different perspectives, expose them to collaborative teamwork, promote engagement and persistence on task, strengthen social relationships, activate team spirit, and facilitate benefits of deep learning through group discussion.

The need for participants to work together to succeed in a time-pressured but fun environment allows students to develop communication skills; it can also reduce the "free-rider" problem (i.e., students who reap the benefits of, but do not contribute to, the group's work), which is one of the common complaints among both instructors and students regarding traditional group-based learning activities. Additionally, escape rooms provide a platform for bringing technology to the classroom, as websites, videos, or other interactive digital material can easily be incorporated into the various puzzle of the escape activity.

Creating puzzles that address the learning objectives and force students to engage with the material instead of just searching for clues requires time and thought. Once the game has been developed though, it can be used repeatedly in successive years. Any implementations of educational escape rooms should include pilot testing in order to estimate the time required to complete the game and to identify any errors that could prevent successful completion.

Throughout the students' participation in the escape room, the debriefing stage is their only opportunity for reflection; without reflection, experience cannot lead to long-term learning. Surprisingly, only $27(39.7 \%)$ of the reported studies included debriefing in their escape room experience and only 15 (22.1\%) provided details of its 
implementation. Further studies incorporating debriefing and with more rigorous evaluation are needed to confirm the educational value for different designs, different types of content, and in different settings. Additional research is also necessary to begin to gauge the "return of investment" of time and resources in relation to the achieved outcomes.

In conclusion, the present review intends to contribute to instructional education design by providing evidence of escape rooms' potential to support teaching and learning across different disciplines. The results may extend the road map for further research, offer new insights to researchers, and provide educators with effective advice and suggestions on how to incorporate escape rooms into their practice.

\section{References}

Adams, V., Burger, S., Crawford, K., and Setter, R. (2018) “Can You Escape? Creating an Escape Room to Facilitate Active Learning", Journal for Nurses in Professional Development, Vol. 34, No. 2, E1YE5.

Barata, G., Gama, S., Jorge, J., and Goncalves, D. (2013) "Engaging Engineering Students with Gamification", In: Proc. of 5th Int. Conf. on Games and Virtual Worlds for Serious Applications (VS-GAMES), Bournemouth, UK, 11-13 Sep 2013, pp. 1-8.

Bartlett, K.A. and Anderson, J.L. (2019) "Using an Escape Room to Support the Learning of Science Content", In: Proceedings of SITE 2019, Las Vegas, NV, United States, March 18-22, 2019, pp. 710-715.

Borrego, C., Fernández, C., Blanes, I., and Robles, S. (2017) "Room escape at class: escape games activities to facilitate the motivation and learning in computer science", Journal of Technology and Science Education, Vol. 7, No. 2, pp. 162-171.

Clarke, S., Peel, D., Arnab, S., Morini, L., Keegan, H., and Wood, O. (2017) “EscapED: A Framework for Creating Educational Escape Rooms and Interactive Games to For Higher/Further Education", International Journal of Serious Games, 4(3), pp. 73-86.

Cohen, J. (1968) "Weighted kappa: nominal scale agreement provision for scaled disagreement or partial credit", Psychol Bull, Vol. 70, No. 4, p. 213.

Csikszentmihalyi, M. (1990) Flow: the psychology of optimal experience, New York: Harper and Row. Dietrich, N. (2018) "Escape Classroom: The Leblanc Process: An Educational Escape Game”. J. Chem. Educ. 2018956996-999.

Ferreiro-González, M. et al. (2019) "Escape ClassRoom: Can You Solve a Crime Using the Analytical Process?”, J. Chem. Educ., Vol. 96, No. 2, pp. 267-273.

Fotaris, P., Mastoras, T., Leinfellner, R., and Rosunally, Y. (2016) “Climbing Up the Leaderboard: An Empirical Study of Applying Gamification Techniques to a Computer Programming Class", Electronic Journal of eLearning, Vol. 14, No. 2, pp. 94-110.

Friedrich, C., Teaford, H., Taubenheim, A., Boland, P., and Sick, B. (2018) "Escaping the professional silo: an escape room implemented in an interprofessional education curriculum", Journal of Interprofessional Care. Foster, T., and Warwick, S. (2018) "Nostalgia, gamification and staff development - moving staff training away from didactic delivery", Research in Learning Technology 2018, 26: 2021.

Giang, C., Chevalier, M., Negrini, L., Peleg, R., Bonnet, E., Piatti, A., and Mondada, F. (2018) “Exploring Escape Games as a Teaching Tool in Educational Robotics", In: Proceedings of the International Conference Educational Robotics 2018 (EDUROBOTICS), Rome, Italy, 11 Oct 2018.

Guo, Y., Goh, D., and Luyt, B. (2017) "Tertiary students' acceptance of a game to teach information literacy", Aslib Journal of Information Management, Vol. 69, No. 1, pp. 46-63.

Hermanns, M. et al. (2017) Using an "Escape Room" toolbox approach to enhance pharmacology education, Journal of Nursing Education and Practice, Vol. 8, No 4.

Ho, A.M. (2018) "Unlocking Ideas: Using Escape Room Puzzles in a Cryptography Classroom", PRIMUS, DOI: 10.1080/10511970.2018.1453568

Hsu, S.-H., Cheng, S.-C., and Huang, Y.-M. (2009) "The Experience of Adopting Game-Based Learning in Library Instruction", In: M. Chang et al. (Eds.): Edutainment 2009, LNCS 5670, pp. 571-576.

Kinio, A., Dufresne, L., Brandys, T., and Jetty, P. (2017) "Break out of the classroom: The use of escape rooms as an alternative learning strategy for surgical education", Journal of Vascular Surgery, Vol. 6, No., e76 Kitchenham, B.A. (2007) "Guidelines for performing Systematic Literature Reviews in Software Engineering Version 2.3", EBSE Technical Report, Keele University and University of Durham.

Kroski, E. (2019) Escape Rooms and other immersive experiences in the library, Chicago: ALA Editions. López-Pernas, S., Gordillo, A., Barra, E., and Quemada, J. (2019) “Examining the use of an educational escape room for teaching programming in a higher education setting", IEEE Access 7, pp.31723-31737. 
Mac Gregor, M. (2018) "Campus Clue: Habituating Students to the Information Search Process via Gaming", Pennsylvania Libraries: Research \& Practice, Vol. 6, No. 2 (Fall 2018), DOI: 10.5195/palrap.2018.172

Moher, D., Liberati, A., Tetzlaff, J., and Altman, D. G. (2009) "Preferred reporting items for systematic reviews and meta-analyses: The PRISMA statement", PLoS Medicine, Vol. 6, No. 7, e1000097.

Nicholson, S. (2015) "Peeking behind the locked door: A survey of escape room facilities", White Paper, pp. 135, [online], http://scottnicholson.com/pubs/erfacwhite.pdf

Peleg, R., Yayon, M., Katchevich, D., Moria-Shipony, M., and Blonder, R. (2019) “A Lab-Based Chemical Escape Room: Educational, Mobile, and Fun!", Journal of Chemical Education, Vol. 96, No. 5, pp. 955-960.

Pellas, N., Fotaris, P., Kazanidis, I., and Wells, D. (2018) "Augmenting the learning experience in primary and secondary school education: A systematic review of recent trends in augmented reality game-based learning", Virtual Reality. Special Issue: VR in Education.

Pun, R. (2017) "Hacking the Research Library: Wikipedia, Trump, and Information Literacy in the Escape Room at Fresno State", Library Quarterly: Information, Community, Policy, Vol. 87, No. 4, pp. 330-336.

Rouse, W.L. (2017) "Lessons learned while escaping from a zombie: designing a breakout edu game", The History Teacher, Vol. 5, No. 4.

Sanchez, E., and Plumettaz-Sieber, M. (2019) "Teaching and Learning with Escape Games from Debriefing to Institutionalization of Knowledge", In: M. Gentile et al. (Eds.): GALA 2018, LNCS 11385, pp. 242-253.

Sundsbø, Katrine (2019) “Open Access Escape Room: The Key to OA Engagement?", Insights, Vol. 32, No.1: 8. Schaffhauser, D. (2017) "Breakout! Gaming to learn", TH E Journal, Vol. 44, No. 4, pp. 6-11.

Serdyukov, P. (2017) “Innovation in education: what works, what doesn't, and what to do about it?", Journal of Research in Innovative Teaching \& Learning, Vol. 10, No. 1, pp. 4-33.

UNESCO (2013) International standard classification of education. Fields of education and training 2013 (ISCED-F 2013) - Detailed field descriptions, DOI: 10.15220/978-92-9189-179-5-en

Vygotsky, L.S. (1978) Mind in Society: The Development of Higher Psychological Processes. Cambridge, MA: Harvard University Press.

Walsh, B., and Spence, M. (2018) "Leveraging Escape Room Popularity to Provide First-Year Students with an Introduction to Engineering Information", In: Proceedings of the Canadian Engineering Education Association (CEEA) Conference, June 3-6, 2018, Vancouver BC.

Zhang, X. C., Diemer, G., Lee, H., Jaffe, R., and Papanagnou, D. (2019) “Finding the 'QR' to Patient Safety: Applying Gamification to Incorporate Patient Safety Priorities Through a Simulated 'Escape Room' Experience", Cureus, Vol. 11, No. 2: e4010, DOI: 10.7759/cureus.4014 